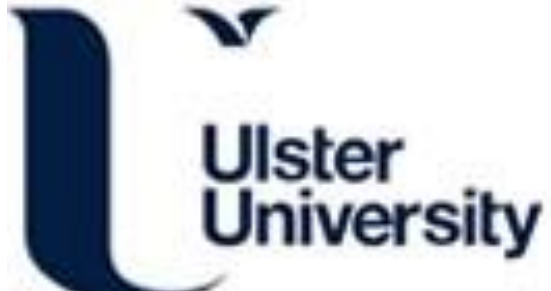

\section{GazeMotive: A Gaze-Based Motivation-Aware E-Learning Tool for Students with Learning Difficulties}

Wang, R., Xu, Y., \& Chen, L. (2019). GazeMotive: A Gaze-Based Motivation-Aware E-Learning Tool for Students with Learning Difficulties. In INTERACT 2019, Lecture Notes in Computer Science (Vol. 11749, pp. 544-548). [Chapter 34] (Human-Computer Interaction - INTERACT 2019; Vol. 11749). Springer Cham. https://doi.org/10.1007/978-3-030-29390-1_34

Link to publication record in Ulster University Research Portal

Published in:

INTERACT 2019, Lecture Notes in Computer Science

Publication Status:

Published (in print/issue): 23/08/2019

DOI:

10.1007/978-3-030-29390-1_34

Document Version

Author Accepted version

\section{General rights}

Copyright for the publications made accessible via Ulster University's Research Portal is retained by the author(s) and / or other copyright owners and it is a condition of accessing these publications that users recognise and abide by the legal requirements associated with these rights.

\section{Take down policy}

The Research Portal is Ulster University's institutional repository that provides access to Ulster's research outputs. Every effort has been made to ensure that content in the Research Portal does not infringe any person's rights, or applicable UK laws. If you discover content in the Research Portal that you believe breaches copyright or violates any law, please contact pure-support@ulster.ac.uk. 


\title{
GazeMotive: A Gaze-based Motivation-aware E-learning Tool for Students with Learning Difficulties
}

\author{
Ruijie Wang ${ }^{1}$, Yuanchen $\mathrm{Xu}^{1}$, and Liming $\mathrm{Chen}^{1}$ \\ ${ }^{1}$ De Montfort University, Leicester, LE1 9BH, UK \\ ruijie.wang@my365.dmu.ac.uk
}

\begin{abstract}
We developed a gaze-based motivation-aware e-learning tool, a Windows desktop learning application, for students with learning difficulties that aims at motivation-enhanced learning by dynamically assessing and responding to students' motivational states based on the motivation model that we developed previously using rigorous methodologies including domain knowledge and empirical studies with participants with learning difficulties. The learning application uses an eye tracker to monitor a user's eye movements during the user's learning process, assesses the user's motivational states using the prediction models we developed before to output personalised feedback from a pedagogical agent in the system based on both the eye gaze features and user's self-input data for enhancing users' motivation and engagement in real-time. Our e-learning tool is an example of applying user modelling and personalisation to an e-learning environment targeting at users' learning motivation, producing great insight on how eye tracking can assist with students' learning motivation and engagement in e-learning environments.
\end{abstract}

Keywords: Eye Tracking, Motivation Assessment, Personalised Feedback.

\section{Introduction}

\subsection{Background}

Students' high level of motivation is fundamental for their learning success. Specifically, students with learning difficulties such as dyslexia can cause low levels of engagement with the education system or dropouts. Thanks to the advancement of assistive learning systems and user modelling techniques for personalised learning, different individual learning needs can be met by personalising learning environments based on user models. User modelling combined with mechanisms of personalisation is necessary to apply the user models to the real-world scenarios and tailor learning service and experience to different individuals' learning needs or mental states. Most of the research pertaining to personalized learning has focused on emotion and cognition of learners such as inducing more positive emotions or reattracting attention; in contrast, e-learning system designers have neglected to apply user modelling and personalized interventions that aims at improvements in exactly learners' motivational states. Therefore, we have developed inference rules based on our motivation model 
developed previously [1] to identify user's motivational states based on the data collected during a learning process including both self-reported data and eye gaze data that can be recorded automatically and finally enable the pedagogical agent to output personalised feedback to user to sustain and enhance motivation in real-time.

\subsection{Motivation Assessment}

Motivation is a multi-faceted concept, and we have developed a conceptual motivation model previously from domain knowledge and interviews as well as a multi-item questionnaire study [1][2]. The model contains factors that determine the continued use intention in an e-learning environment from intrinsic motivation such as Self-efficacy, extrinsic motivation such as Visual Attractiveness, and mediators such as Reading Experience. Eye gaze data has shed light on human's various cognitive process including problem solving [3], and eye gaze features like pupil dilation has been used as indicators of emotional states [4]. In a previous experiment with students with learning difficulties [5], we have found that gaze features such as average pupil diameter and fixation number can play significant roles in assessing learners' motivation in e-learning context with a prediction accuracy up to $81.3 \%$, which can be a good alternative to the approach using merely self-reported data to avoid the selfreported bias.

\subsection{The Demonstration System}

The demonstration system is a gaze-based learning application that assesses a learner's motivation related to the factors in our motivation model mentioned before based on the features computed from both the self-input data and real-time data from the Tobii eye tracker $4 \mathrm{c}$. The system then uses personalization algorithms to generate personalised feedback based on the motivation assessment to address the corresponding motivational needs. Different motivational factors are assessed based on different gaze features with different parameters using the logistic regression models resulted from our previous study [5], starting from which, we have improved the prediction models by including only the gaze features that have significant prediction power. The system implemented some regression models to assess the corresponding motivational factors (e.g., Confirmed Fit and Reading Experience) purely based on gaze features. Other factors such as Attitudes Toward School and Self-efficacy were assessed based on selfreported data collected at the beginning of a learning process, as they involve intrinsic motivation that usually remains stable in a short-term period. The system also outputs personalised feedback at the stage of self-assessment quizzes based on both a user's answer and gaze data. Given that we have developed the motivation model and classification algorithms using rigorous approaches and have evaluated the relevant motivational strategies [5], we have confidence that the present system will yield motivation-enhanced learning experience and better learning performance over the long term by monitoring and incorporating eye tracking it into motivation assessment and providing personalised feedback to respond to different motivational states in real-time. 


\section{GazeMotive Walkthrough}

GazeMotive was designed for two user groups, expert and learner. When a user logs on with a username and a password (see Fig. 1.a), the system will redirect the user to either the expert interface or the learner interface. The expert interface allows an expert user to add or delete learning materials, page by page. The expert user can then input selfassessment quizzes after each lesson (see Fig. 1.b). Any learning materials can be added to our system in front-end by expert users in picture format, and this demonstration system uses materials adapted from a free e-learning course [6], the frozen planet, as an example. We adopted 16-40pt Verdana dark fonts on light yellow background and visual elements like images according to the design principles for students with learning difficulties [7]. Our system assesses learners' motivational states from gaze features, some of which are computed based on AOIs (Areas of Interest), and different learning materials and pages have different AOIs, so expert users need to select one or more AOIs for each page by clicking on the points at the corners of the polygons to enable relevant gaze features to be computed, and an AOI can also be selected for review or deletion by the expert users (see Fig. 1.c and Fig. 1.d).

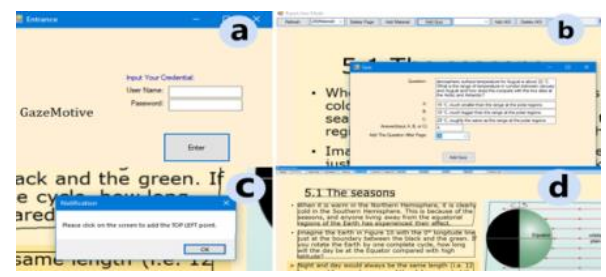

Fig. 1. Screenshots of expert interface showing an example of (a) login, (b) adding a quiz, (c) the process of adding an AOI, and (d) the AOIs added.

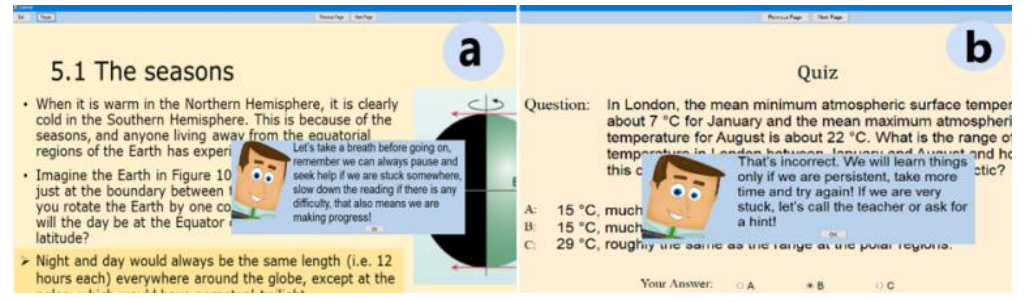

Fig. 2. Screenshots of user interface showing the examples of personalized feedback to provide motivational help (a) when the system detects a user to have negative reading experience from eye-tracking data in a learning page, and (b) when the user submits an incorrect answer to a quiz and is meanwhile detected from the eye-tracking data as having not put enough effort.

The learner interface is similar with the expert one, the main difference is the personalised feedback output from the system to user dependent on the motivational states detected by the system from eye-tracking data or self-reported data or a combination of both. During a learning process, a pedagogic agent representing a virtual tutor will output personalised feedback to address specific motivational needs. 
The feedback implemented in our system is in format of text and picture, and more diverse formats of feedback and interventions such as speech and animation can also be incorporated into the inference rules and implemented following our present work. Fig. 2 shows two examples of personalised feedback based on real-time motivation assessment, in the learning stage (see Fig. 2.a) and the quiz stage (see Fig. 2.b), respectively.

\section{Conclusion}

In this paper, we demonstrate how we have applied the motivation model and corresponding personalisation rules to real-world scenarios. The motivation model and classification algorithms were developed from our previous studies using interviews, a multi-item questionnaire and an experiment with target users. The inference rules and motivational strategies were adapted to our learning environment based on our motivation model. Our system uses eye tracking technology to assess motivational states by monitoring gaze features and self-input data during user's learning process, and this motivation-aware system demonstrated a way of applying the motivational strategies to dynamically respond to the motivational states detected based on our motivation model using gaze data instead of relying on obtrusive self-report data towards accurate real-time motivation assessment for enhanced motivation in an elearning environment. In future, we will keep validating the models and algorithms to maximize the accuracy of motivation assessment and personalised feedback, as well as improving the interface design. Additionally, more dimensions of motivation and more diverse formats of feedback will be implemented using the logic that has been developed in the system.

\section{References}

1. Wang, R., Chen, L., Solheim, I., Schulz, T., Ayesh, A.: Conceptual Motivation Modeling for Students with Dyslexia for Enhanced Assistive Learning. In: SmartLearn'17 Proceedings of the 2017 ACM Workshop on Intelligent Interfaces for Ubiquitous and Smart Learning. pp. 11-18. ACM, Leicester (2017).

2. Wang, R., Chen, L., Solheim, I.: Modeling Dyslexic Students' Motivation for Enhanced Learning in E-learning Systems. Submitted to ACM TiiS.

3. Poole, A., Ball, L.: Eye Tracking in Human-Computer Interaction and Usability Research: Current Status and Future Prospects. Encyclopedia of Human Computer Interaction. 211219 (2006).

4. Alghowinem, S., Alshehri, M., Goecke, R.: Exploring Eye Activity as an Indication of Emotional States Using an Eye-tracking Sensor. In: Chen, L., Kapoor, S. and Bhatia, R. (ed.) Studies in Computational Intelligence. pp. 261-276. Springer Cham (2014).

5. Wang, R., Chen, L., Ayesh, A., Shell, J., Solheim, I.: Gaze-based Assessment of Dyslexic Students' Motivation within an E-learning Environment. Accepted by IEEE UIC 2019.

6. M. Brandon, "The Open University course S175: The frozen planet," http://www.open.ac.uk/courses/modules/s175, last accessed 2019/04/08. 
7. Dyslexic.com, https://www.dyslexic.com/blog/dyslexia-and-visual-stress-what-assistivetechnology-can-help, last accessed 2019/04/08.

\section{Technical requirements}

\section{Hardware}

1. A Tobii Consumer Eye Tracker, such as Tobii Eye Tracker $4 c$

2. A laptop or desktop Windows OS computer

\section{Software}

1. Visual Studio 2017

- We developed the application with Visual Studio 2017. We haven't published the application to be an installation package, so Visual Studio 2017 is used to open the project. Visual Studio will automatically organise the project environment with the .sln file. The application then can be run with Visual Studio.

- Other versions of Visual Studio may occur database connection error. To fix the problem, users using different Visual Studio versions may need to check and change the database connection string that is a static property of the DatabaseConnection class of the project.

2. Tobii.Interaction package with version 0.7 .3

- $\quad$ NuGet package manager of Visual Studio can obtain the package.

3. Tobii Eye Tracking Core Software (https://gaming.tobii.com/getstarted/)

- After installing the software, make sure the "Display setup" is run at least one time to let the eye tracker adapt to the monitor resolution.

- Make sure the Tobii eye tracking is switched to "On" before running the application.

\section{Others}

- If users of the application want to get correct coordinates of the areas of interest when they click on the learning materials through "Expert User Mode", make sure the "Change the size of apps and text on the main display" of Windows OS is set to $100 \%$. 